\title{
Broadening our understanding of genetic risk for scleroderma/systemic sclerosis by querying the chromatin architecture surrounding the risk haplotypes
}

Kerry E. Poppenberg ${ }^{1,2}$, Vincent M. Tutino ${ }^{1,2,3,4}$, Evan Tarbell ${ }^{5}$ and James N. Jarvis J,6,7* $^{*}$

\begin{abstract}
Background: Genetic variants in the human leukocyte antigen (HLA) locus contribute to the risk for developing scleroderma/systemic sclerosis (SSc). However, there are other replicated loci that also contribute to genetic risk for $\mathrm{SSC}$, and it is unknown whether genetic risk in these non-HLA loci acts primarily on the vasculature, immune system, fibroblasts, or other relevant cell types. We used the Cistrome database to investigate the epigenetic landscapes surrounding 11 replicated SSc associated loci to determine whether SNPs in these loci may affect regulatory elements and whether they are likely to impact a specific cell type.

Methods: We mapped 11 replicated SNPs to haplotypes and sought to determine whether there was significant enrichment for H3K27ac and H3K4me1 marks, epigenetic signatures of enhancer function, on these haplotypes. We queried pathologically relevant cell types: B cells, endothelial cells, fibroblasts, monocytes, and T cells. We then identified the topologically associated domains (TADs) that encompass the SSc risk haplotypes in primary T cells to identify the full range of genes that may be influenced by SSc causal SNPS. We used gene ontology analyses of the genes within the TADs to gain insight into immunologic functions that might be affected by SSc causal SNPS.

Results: The SSc-associated haplotypes were enriched ( $p$ value $<0.01$ ) for H3K4me1/H3K27ac marks in monocytes. Enrichment of one of the two histone marks was found in B cells, fibroblasts, and T cells. No enrichment was identified in endothelial cells. Ontological analyses of genes within the TADs encompassing the risk haplotypes showed enrichment for regulation of transcription, protein binding, activation of T lymphocytes, and proliferation of immune cells.

Conclusions: The 11 non-HLA SSc risk haplotypes queried are highly enriched for H3K4me1/H3K27ac-marked regulatory elements in a broad range of immune cells and fibroblasts. Furthermore, in immune cells, the risk haplotypes belong to larger chromatin structures encompassing genes that regulate a wide array of immune processes associated with SSC pathogenesis. Though importance of the vasculature in the pathobiology of SSc is widely accepted, we were unable to find evidence for genetic influences on endothelial cell function in these regions.
\end{abstract}

Keywords: Scleroderma, Systemic sclerosis, Genetic risk, Histone mark, Topologically associated domain

*Correspondence: jamesjar@buffalo.edu

${ }^{5}$ Quantitative Systems Pharmacology, Enhanced Pharmacodynamics,

LLC, Buffalo, NY, USA

Full list of author information is available at the end of the article

\section{Background}

Scleroderma, or systemic sclerosis (SSc), is a spectrum of human diseases characterized by sclerosis involving multiple organs, most prominently the skin and gastrointestinal tract [1], and is accompanied by distinct 
patterns of autoantibody production [2]. SSc is frequently accompanied by clinically-prominent vascular manifestations such as Raynaud's phenomenon [3, 4], as well as prominent signs of microvascular injury to a broad range of tissues including the heart and lungs [5]. These clinical manifestations, in addition to multiple lines of clinical and basic research, suggest a complex pathogenesis involving interactions between the immune system, the microvasculature, and fibroblasts [6]. The question remains as to which of these three different features is the primary driver of the disease. Answering this question is of considerable importance, as it would facilitate the search for new and effective therapies.

One way of gaining insight into whether the immune, fibrotic, or vascular phenomena are primary in SSc pathogenesis is to determine where genetic effects that confer risk for the disease are exerted, i.e., vasculature, the immune system, or fibroblasts. Genetic variants have long thought to contribute to the risk for SSc. For example, associations between the human leukocyte antigen (HLA) locus and SSc are well recognized [7]. Furthermore, while SSc disease prevalence is quite low (50-300 cases per million or no more than $0.03 \%$ of the population), the disease prevalence in siblings of affected individuals may be as high as $2.6 \%$ [8]. Several genome-wide association studies (GWAS) have been performed on patients with SSc, and, predictably, the HLA locus has been replicated as the most important region for genetic risk in all of these studies [9-12]. While these data provide evidence that the immune system may be a primary driver of the clinical entity we call SSc, there are at least 11 other loci outside of the HLA locus that have been replicated in at least 2 independent studies [8]. We therefore asked whether there was any evidence that genetic effects at these loci might be exerted on vascular, rather than immune cells.

To accomplish this task, we used an approach similar to that taken in our previously-reported investigations of juvenile idiopathic arthritis [13], systemic lupus [14], and intracranial aneurysm [15]. We queried the 11 replicated, non-HLA loci associated with SSc to determine whether these loci were enriched for regulatory elements that are generally thought to be involved in autoimmune disease risk [16, 17], mining publicly available data on a variety of related cell types. Furthermore, we examined the broader chromatin architecture surrounding the SSc risk haplotypes to gain further insight into the mechanisms through which variants in these 11 haplotypes might confer disease risk.

\section{Methods}

\section{Defining LD blocks}

We queried 11 single nucleotide polymorphisms (SNPs) in non-HLA loci with established and replicated associations with SSc identified by GWAS and genetic fine mapping studies and reported in Korman et al. [8]. We then used the SNiPA online single nucleotide polymorphism annotator [18] (https://snipa.helmholtz-muenchen.de/ snipa3/) to define linkage disequilibrium (LD) blocks for each of the 11 SNPs. We used the following settings: GRCh37, 1000 Genomes Phase 3 v5, querying European populations, and setting $\mathrm{r}^{2}$ at 0.8 . The smallest genomic position was used as the start of the LD block while the largest position was used as the end of the block. The liftover tool from UCSC was used to convert the positions to GRCh38. These regions are presented in Table 1.

\section{Identification of H3K4me1/H3K4me3/H3K27ac histone marks within LD blocks}

In the 11 risk haplotypes, we assessed the presence of H3K4me1 and H3K27ac histone marks, which typically identify weak and strong enhancers respectively, within the 11 risk haplotypes. We also examined H3K4me3 marks to identify promoters. We queried H3K4me1/ H3K4me3/H3K27ac ChIPseq data available from the Cistrome database (http://cistrome.org/db/\#/) from multiple cell types: CD19+ B lymphocytes, fibroblasts, human umbilical vein endothelial cells (HUVECs), CD14+ monocytes, and $\mathrm{CD} 4+\mathrm{T}$ lymphocytes. The Cistrome dataset information is provided in Table 2 .

We used the intersect command within the BEDTools software package to identify intersections between H3K4me1/H3K4me3/H3K27ac peak data and linkage

Table 1 Positional information of the 11 scleroderma-risk single nucleotide polymorphisms and the associated haplotypes

\begin{tabular}{llll}
\hline SNP & Haplotype & SNP location & Locus name \\
\hline rs2205960 & chr1:173222336-173287411 & Intergenic & TNFSF4 \\
rs10488631 & chr7:128945562-129071820 & Intergenic & IRF5 \\
rs11642873 & chr16: 85956661-85958099 & Intergenic & IRF8 \\
rs2056626 & chr1:167448647-167467063 & Intronic & CD247 \\
rs3790567 & chr1:67340123-67361333 & Intronic & IL12RB2 \\
rs3821236 & chr2:191035723-191071078 & Intronic & STAT4 \\
rs77583790 & chr3:159907604-159976265 & Intronic & SCHIP1-IL12A \\
rs9373839 & chr6:106181815-106339294 & Intronic & ATG5 \\
rs5029939 & chr6:137849452-137921300 & Intronic & TNFAIP3 \\
rs1378942 & chr15:74751897-74821981 & Intronic & CSK \\
rs2305743 & chr19:18068862-18092777 & Intronic & IL12RB1 \\
\hline
\end{tabular}

SNPs from Korman et al. [8] were mapped to haplotype blocks using SNiPA. SNP location was determined using the UCSC Genome Browser

chr chromosome, rs reference SNP, SNP single nucleotide polymorphism 
Table 2 Cistrome dataset information

\begin{tabular}{lllll}
\hline Publication & Cell type & Cell population & Factor & GEO accession \\
\hline Kim DE, 2017 & Fibroblast & Primary dermal fibroblasts & H3K27ac & GSM2151910 \\
Adoue V, 2014 & Fibroblast & Fibroblast primary cell line & H3K4me1 & GSM1435528 \\
Adoue V, 2014 & Fibroblast & Fibroblast primary cell line & H3K4me3 & GSM1435529 \\
Andersson R, Nature 2014 & B Lymphocyte & CD19+ & H3K27ac & GSM998997 \\
Andersson R, Nature 2014 & B Lymphocyte & CD19+ & H3K4me1 & GSM998994 \\
Bernstein BE, Nat. Biotechnol 2010 & B Lymphocyte & CD19+ & H3K4me3 & GSM1027300 \\
Andersson R, Nature 2014 & T Lymphocyte & CD4+ (strain naïe) & H3K27ac & GSM999004 \\
Andersson R, Nature 2014 & T Lymphocyte & CD4+(strain naïve) & H3K4me1 & GSM999003 \\
LaMere SA, Genes Immun 2016 & T Lymphocyte & CD4+ (strain naïve) & H3K4me3 & GSM1888744 \\
ENCODE Project Consortium, Nature 2012 & Endothelial Cell & HUVEC & H3K27ac & GSM733691 \\
ENCODE Project Consortium, Nature 2012 & Endothelial Cell & HUVEC & H3K4me3 & GSM733690 \\
ENCODE Project Consortium, Nature 2012 & Endothelial Cell & HUVEC & H3K27ac & GSM1003559 \\
ENCODE Project Consortium, Nature 2012 & Monocyte & CD14+ & H3K4me1 & GSM1003535 \\
ENCODE Project Consortium, Nature 2012 & Monocyte & CD14+ & H3K4me3 & GSM1003536 \\
ENCODE Project Consortium, Nature 2012 & Monocyte & CD14+ & &
\end{tabular}

disequilibrium LD regions (haplotypes) for each cell type, as described by Jiang et al. [19]. To determine significance, we created 11 random regions with length equal to the average length of the 11 LD blocks of interest (59,976 bp). Using bedtools intersect, we determined how many random regions overlapped with the peak file for a given cell type and H3K4me1/H3K4me3/H3K27ac enrichment. We repeated this process 1000 times to approximate a normal distribution. We then determined where the number of overlaps with the regions of interest fell within the normal curve in order to calculate the associated $p$ value. Enrichment for histone post-translational modifications (H3K4me1/H3K4me3/H3K27ac) showing a $p$ value $<0.01$ was considered statistically significant.

We also examined expression of locus genes for cell types with significant enrichment for both enhancer marks using primary cell RNA sequencing data. Genes were considered expressed if they had TPM $>1$ in at least half of samples analyzed.

\section{Identification of relevant topologically associated domains in $\mathrm{CD} 4+\mathrm{T}$ cells}

We next sought to identify potential gene targets of H3K4me1/H3K27ac-marked enhancers on the SSc-associated risk haplotypes. While enhancers may not regulate the nearest gene, they typically regulate genes within the same chromatin loop or topologically associated domain (TAD) [20-22]. We used previously published CTCFHiChIP data from primary CD4+ $\mathrm{T}$ cells to identify CTCF loop anchors within the SSc risk haplotypes [23]. Although these data were generated in pediatric samples, they provide detailed view of 3D chromatin structures in a relevant primary human cell. HiChIP loops were converted into paired end BED files; only loops supported by two or more reads were retained. If an LD block did not contain a CTCF loop anchor, it was considered inactive in CD4+ T cells and excluded. If an LD block did contain a CTCF loop anchor as identified on ChIPseq performed on the same samples, we determined all genomic regions that physically interacted with the block. We then identified any regions that interacted with those anchors, and continued this process until all regions demonstrating close physical proximity with the LD block were identified. Further, we used bedtools merge to merge all anchors with a maximum gap between anchors of $1 \mathrm{MB}$ to define positions for the associated TADs. Figure 1 illustrates an example of CTCF loops and binding surrounding TNFAIP3 locus. Next, we used the UCSC genome browser to identify genes within the TADs of the 11 replicated SSc-associated risk haplotypes. We determined whether these genes were expressed in healthy control CD4+ cells at level of TPM $>1$ in at least $50 \%$ of samples. Only genes found to be expressed in CD4+ were used for ontological analyses.

Due to lack of primary cell data, we took a different approach to identify TADs in monocytes. We used the publicly available Juicebox software [24] to identify TADs in THP-1 monocytes using data from Phanstiel et al. [25]. We applied the balanced normalization to each $\mathrm{Hi}-\mathrm{C}$ map to correct for any experimental bias, per author's recommendation [24]. We used a $5 \mathrm{~KB}$ resolution to visualize chromatin loops when identifying SSc TADs. We loaded RefSeq genes via the 1D annotation panel to determine which genes were encompassed within the TAD. We used the straight edge tool to precisely locate 


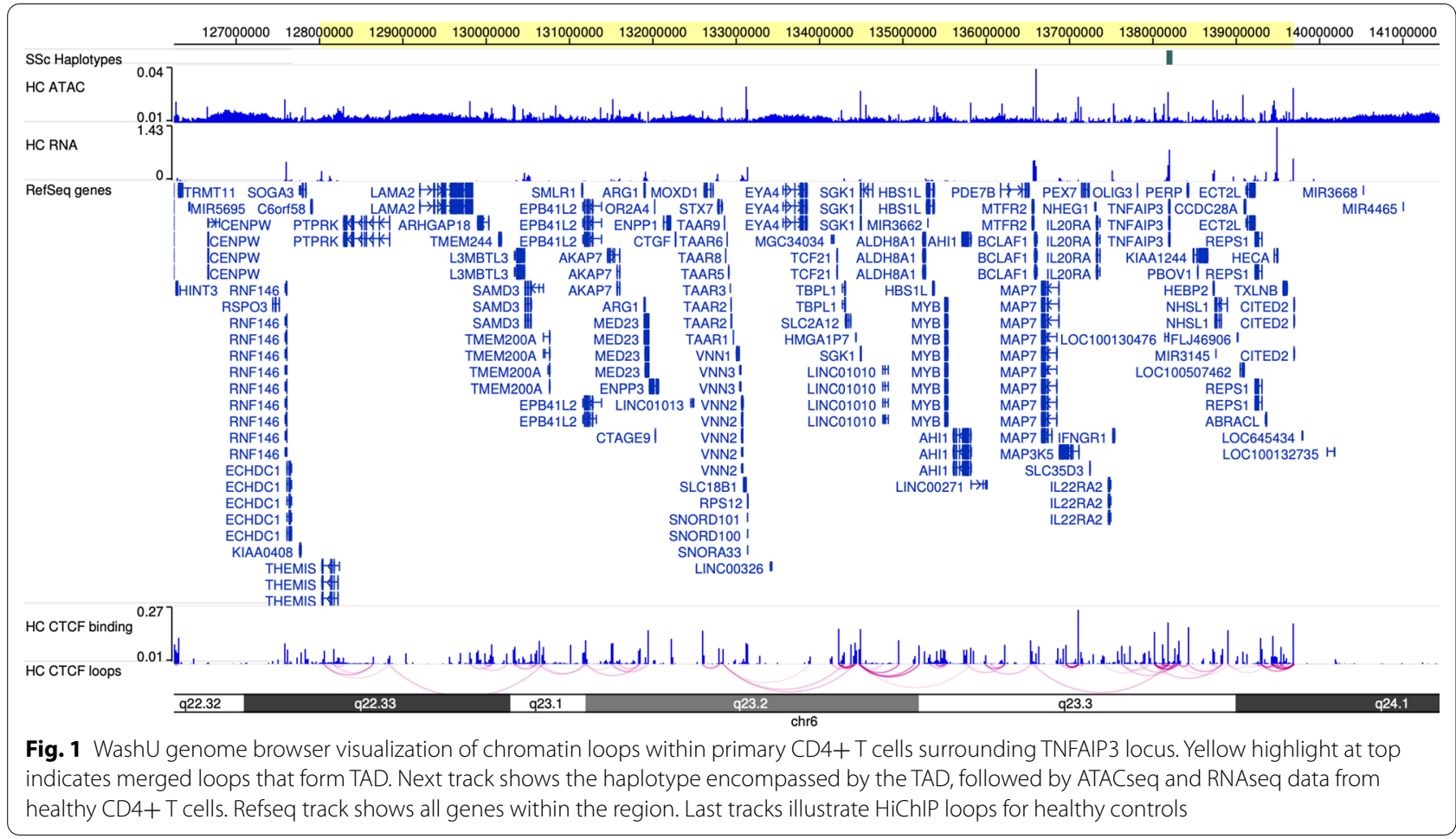

the haplotype and then to identify the exact chromosomal positions of the TAD as displayed in the information pane. As TADs are defined visually, which may differ from user to user, we verified our analyses by querying monocyte CTCF ChIP-seq data downloaded from Cistrome under accession number GSM1003508 (Liftover tool was used to convert TAD coordinates from Juicebox from hg19 to hg38 to match CTCF data) to assure that the identified TADs had the predicted CTCF anchors on each side. Figure 2 shows an example of the landscape around TNFAIP3; the upper portion depicts the TAD defined in Juicebox and the lower browser window shows the CTCF peaks along with the TAD boundaries. The TADs with CTCF peaks at the boundaries were considered to be true TADs, and the genes encompasses within the TAD were recorded. CTCF peaks within the TAD structure represent subloops. As done with $\mathrm{T}$ cells, genes with expression level of TPM $>1$ in at least $50 \%$ of healthy monocyte samples were used for ontological analyses.

\section{Identification of ontologies and functions of genes within TADs}

We performed gene ontology enrichment analysis on expressed genes within TADs in CD4+ $\mathrm{T}$ cells compared against a background of all protein coding genes, obtained from biomart ensembl, using the public Gene Ontology enRIchment anaLysis and visuaLizAtion tool (GORILLA) [26]. We further used Reduce Visualize Gene
Ontology (REVIGO) tool to reduce ontologies based on semantic similarity measures [27]. To better understand potential mechanisms associated with these potential target genes, we used Ingenuity Pathway Analysis (IPA) to identify top disease and biological functions [28]. Functions with a Benjamini-Hochberg $p$ value $<0.01$ that corresponded to 5 or more input genes were reported.

\section{Results}

\section{H3K27ac and H3K4me1 enrichment within the SSC LD} blocks

We used Cistrome H3K27ac and H3K4me1 ChIPseq data to query the SSc-associated LD blocks to determine whether the disease-associated haplotypes are enriched (compared to randomly-selected regions of the genome) for these functional marks in pathologically relevant cell populations. We also queried presence of H3K4me3 marks. Table 3 reports the presence or absence of H3K27ac, H3K4me1, and H3K4me3 marks in the $11 \mathrm{LD}$ blocks of interest for all cell types. These marks can be visualized as individual tracks for the cells queried in the UCSC Genome Browser. For example, Fig. 3 depicts the chromatin landscape surrounding rs5029939 in which we can see histone marks present within the haplotype. Landscape figures for other loci are provided in Additional file 1.

While both H3K4me1/H3K27ac marks were found in the majority of LD blocks for all cell types, only 


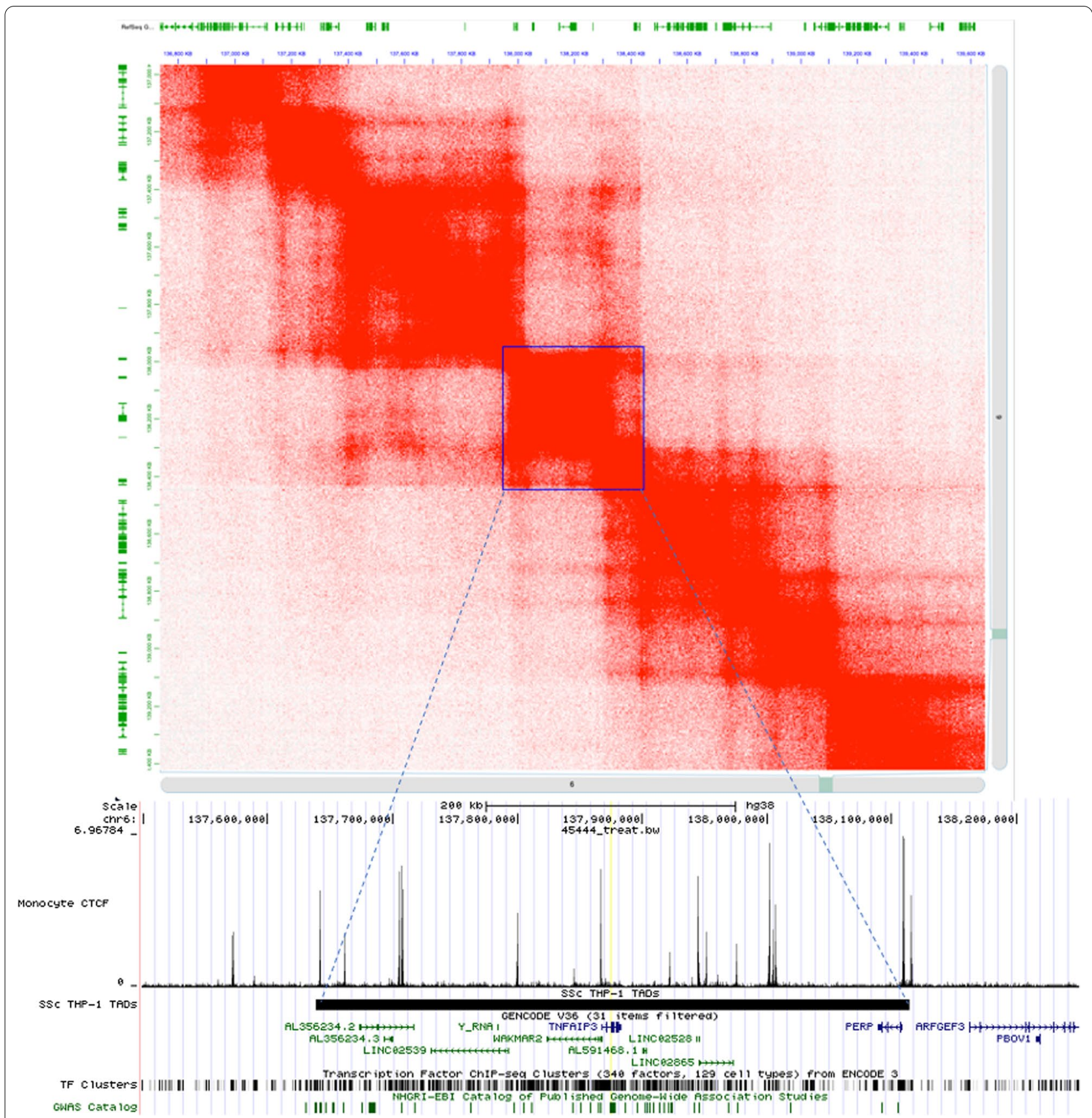

Fig. 2 Juicebox defined TAD for the TNAIP3 locus with CTCF ChIP-seq data in monocytes to corroborate TAD boundaries. HiC map of THP-1 cells used to identify TAD (blue box). Green lines at top and left represent RefSeq genes. TAD coordinates compared to monocyte CTCF peaks to verify if TAD defined correctly

monocytes showed significant enrichment by empirical p-value calculation for both epigenetic marks $(p=0.003$ for H3K27ac, $p=0.004$ for H3K4me1) compared to the background. It is important to note that H3K4me3 marks were also significantly enriched within the haplotypes in monocytes $(p=0.010)$, and this enrichment may reflect genetic influences on promoters in these cells. We found that 10 of 11 locus genes (excluding SCHIP-IL12A) were expressed in monocytes when querying publicly available RNA sequencing data (GSE147608, $\mathrm{n}=11$ ). We also identified significant enrichment for H3K27ac marks (but not H3K4me1) in B cells $(p=0.003)$ and CD4+ T cells $(p=0.007)$, and significant enrichment for H3K4me1 


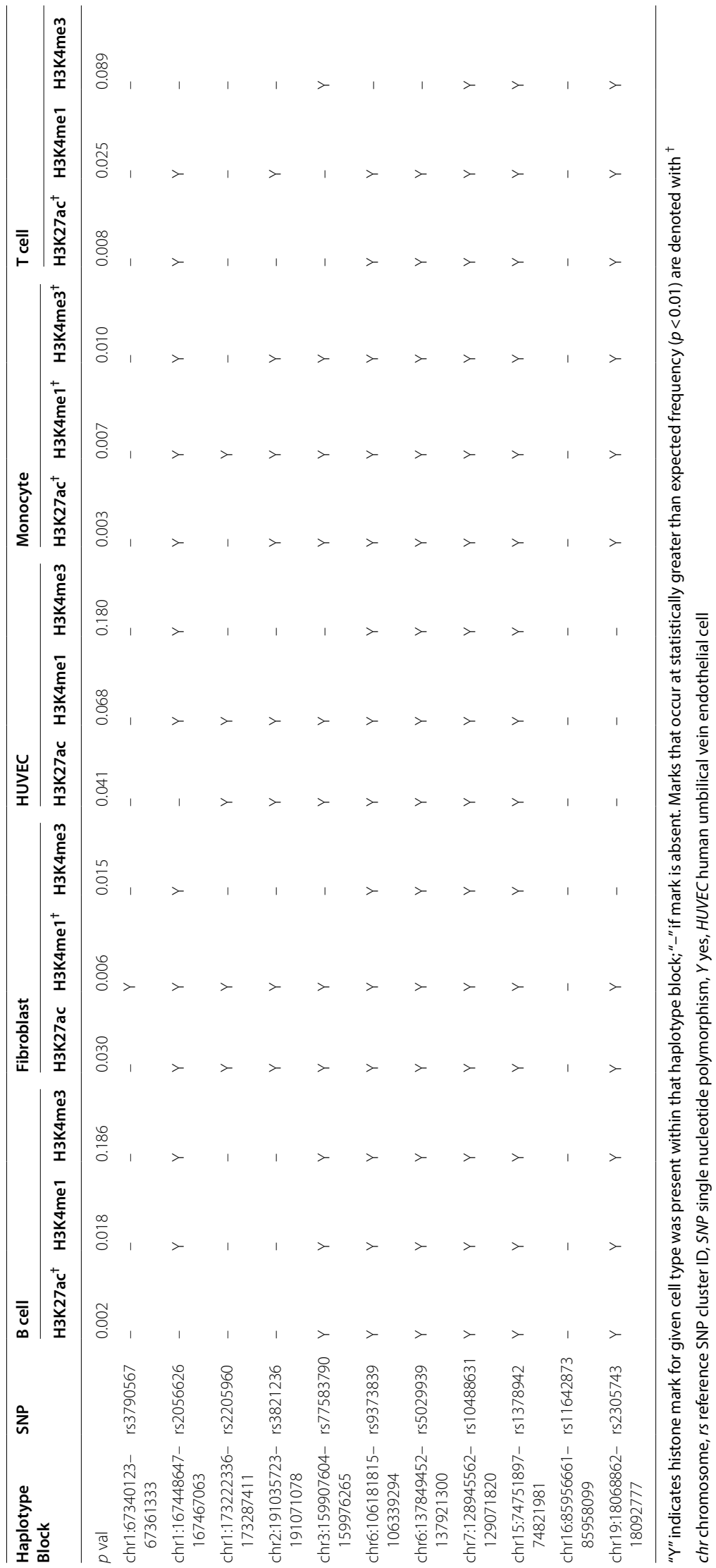




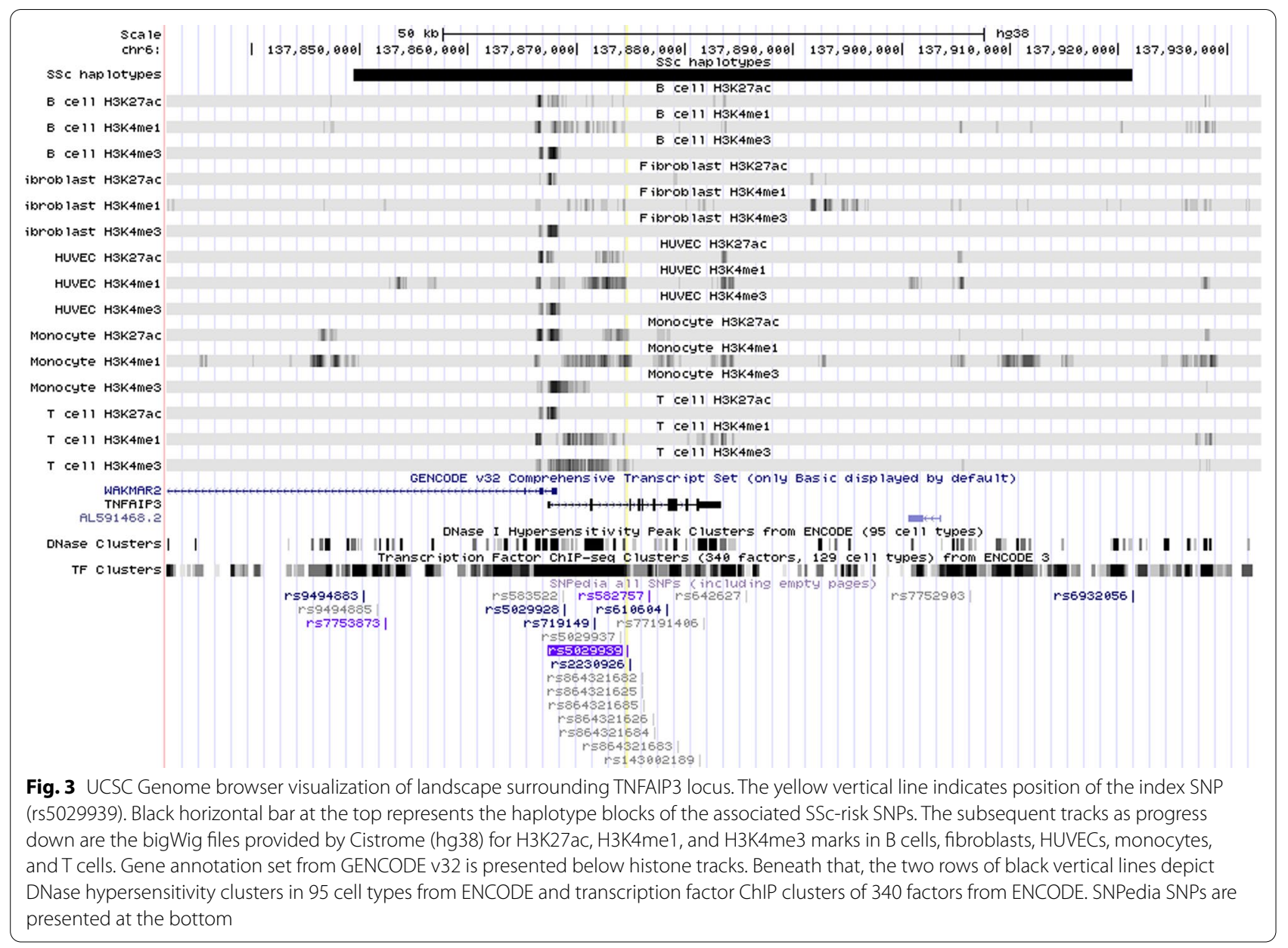

(but not H3K27ac) marks in fibroblasts $(p=0.008)$. Neither mark was enriched in HUVECs.

\section{Ontological analysis of genes within TADs of CD4+ T cells} H3K4me1/H3K27ac histone marks identify regions that have a strong likelihood of exhibiting enhancer function. However, enhancers do not always regulate the most proximal gene (in terms of linear genomic distance). Gasperini et al. [22] have shown that $>70 \%$ of genes regulated by enhancers lie within the same TAD as the enhancers, and that regulatory effects outside the TAD are generally weaker than those within the TAD.

First, we examined T cells using HiChIP-defined TADs from CD4+ primary cell data. CTCF loop anchors were not present in 4 of the SSc-associated haplotypes. For the haplotypes in which CTCF anchors were present, we identified a total of 854 genes. Of those genes, 502 were expressed in $\mathrm{CD} 4+\mathrm{T}$ cells of healthy individuals. We then identified 41 associated biological processes, including regulation of transcription, protein transport, and regulation of metabolic process. A reduced list of these terms generated using REVIGO is presented as a treemap in Fig. 4a. Next, we surveyed molecular functions and found 21 significant functions associated with the genes expressed within the relevant $\mathrm{CD} 4+\mathrm{T}$ cells TADs. These functions included transcription regulator activity, DNA binding, protein binding. Additional file 2 presents the full list of GORILLA results. We further used IPA to examine top diseases and biological functions for the refined set of genes that were expressed in CD4+ T cells. We used a Benjamini-Hochberg $p$ value $<0.01$ and the requirement that at least five of the input genes were assigned to the annotation to identify significant terms. There were 92 annotations reported, including activation of $\mathrm{T}$ lymphocytes, proliferation of immune cells, transcription of RNA, and binding of DNA. See Additional file 3 for full significant results from IPA.

Next, we investigated the ontologies associated with genes expressed within monocyte TADs. All 11 TADs defined visually in Juicebox also had CTCF peaks present at those boundaries and encompassed 113 genes, of which 59 genes were expressed in healthy 


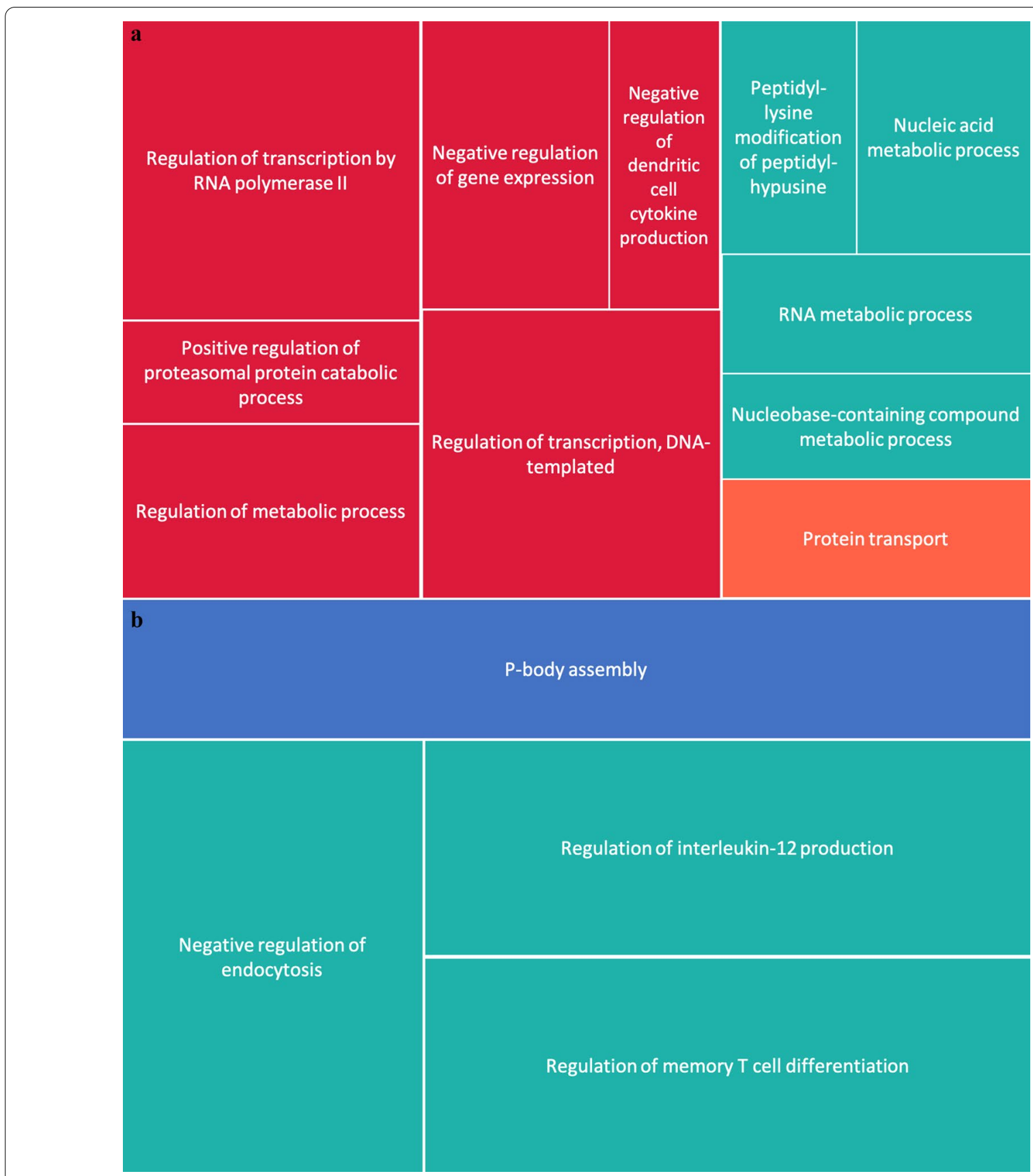

Fig. 4 REVIGO treemaps of biological processes associated with expressed genes within CD4+ and monocyte TADs surrounding SSC loci. a Reduced biological processes for genes expressed within CD4+TADs. b Reduced biological processed for genes expressed within monocyte TADs

monocytes. These genes associated with 19 biological processes, predominantly focused on cytokine production and signaling. The reduced terms identified by REVIGO are presented in a treemap in Fig. 4b. Only one molecular function, RNA polymerase II core promoter sequence-specific DNA binding, was identified.
Full lists for associated biological processes and molecular functions are provided in Additional file 2. Using IPA, we identified 43 significant disease and biological functions, including activation of lymphocytes, systemic lupus erythematosus, and cytotoxicity of cells. The full list is presented in Additional file 3. 


\section{Discussion}

It is becoming increasingly clear that, for most complex genetic traits, including autoimmune diseases, genetic risk is largely exerted on regulatory elements that control gene expression rather than on the protein-coding sequences of relevant genes [16, 29]. These regulatory regions, and their most likely target genes, can be identified based on specific features of the surrounding chromatin. Although these features are not prima facie evidence that the region of interest has regulatory function [21], they can be useful guides to assess probable genetic mechanisms. Therefore, in this study we used the broader chromatin architecture encompassing the established and replicated SSc haplotypes as a means of investigating the mechanisms through which genetic variants might confer disease risk.

Using ChIPseq data accessed through the Cistrome database, we queried H3K27ac/H3K4me1 enrichment in 11 replicated SSc risk haplotypes in multiple pathologically relevant cell types: B cells, fibroblasts, HUVECs, monocytes, and $\mathrm{T}$ cells. We found evidence for genetic influences on regulatory regions in immune cells, particularly monocytes (enriched for both H3K27ac and H3K4me1) and fibroblasts (enriched for H3K4me1, which typically marks poised rather than active enhancers), and CD4+ T cells (enriched for H3K27ac, a marker for active enhancers). However, we found no evidence that genetic risk operates on the vasculature, as neither epigenetic mark was significantly enriched in HUVEC compared with genome background. Furthermore, examining expressed genes found in the TADs that encompass the SSc risk haplotypes using primary $\mathrm{CD} 4+$ data, we identified multiple expression related cellular processes (e.g., regulation of gene expression, regulation of transcription by RNA polymerase II, protein transport).

Examining the IPA-annotated disease and biological functions in $\mathrm{CD} 4+\mathrm{T}$ cells, we found proliferation of immune cells and activation of $\mathrm{T}$ lymphocytes were among the significant, further corroborating the pathologic relevance of these analyses. Additional refinement of these data, for example, by performing gene expression experiments in a well-characterized and genotyped population, may be a promising way to identify previously unsuspected targets of therapy for SSc. We also note that cancer is one of the most significant disease categories that emerged from the IPA analysis of the genes in CD4+ $\mathrm{T}$ cells TADs. There is an increased risk of malignancy in patients with SSc and some suggest that SSc is related to an immune response against cancer [30]. Gene profiling by Dolcino et al. also reflected an oncogenic gene signature [31]. We note 38 of the genes found expressed in $\mathrm{T}$ cells TADs encompassing the scleroderma associated SNPs were also found to be differentially expressed in Dolcino's study [31], including BSG, FCER2, GPA33, MAP2K7, SCAMP2, and TSPAN33.

It is interesting that neither H3K4me1 nor H3K27ac is enriched in the SSc haplotypes in HUVECs, given the abundant evidence that the microvasculature is the primary target of injury in this disease. One of first clinical signs of SSc is Raynaud's phenomenon, the loss of normal regulation of cutaneous vessels, which affects thermoregulation. The areas most afflicted by impaired thermoregulation, predominantly the extremities, are also the regions with most progressed skin fibrosis [5]. Raynaud's phenomenon is thought to result from endothelial injury. Another clinical sign of SSc is abnormal capillary structure, which reflects microvascular damage that may be due to both neovascularization and loss of vascularization due to impaired blood flow [32]. Following microvascular injury, as the disease progresses, there is further involvement of endothelium and blood vessels within multiple organs (heart, lungs, kidneys, and GI tract), resulting in tissue ischemia, fibrosis, and organ failure [5]. Vascular remodeling and subsequent tissue fibrosis are the next stages in pathogenesis, which further suggests that the vasculature is the main target in SSc. However, our data do not allow us to conclude that genetic influences are the primary driver of these well-documented vascular phenomena in SSc.

A novel finding in this study is the implication of monocytes, which were highly enriched for H3K4me1/ H3K27ac marks within the SSc risk haplotypes. It is known that monocytes are among the infiltrates following endothelial injury in SSc [33]. Monocytes can produce fibroblasts [34], which are critical in the progressive fibrosis indicative of scleroderma, and can differentiate into macrophages, an important cell in tissue homeostasis and inflammation [35]. Both cell types have reported abnormalities in scleroderma [36, 37]. It has also been suggested that there is a profibrotic phenotype expressed by circulating monocytes in SSc patients [38]. Monocytes have been associated with fibrotic disease; for instance, elevated monocyte count has been reported as a biomarker for poor outcome [39, 40]. We also found H3K4me3 marks to be enriched on the SSc haplotypes in monocytes, which, as we noted previously, may reflect genetic influences on active promoters in these cells. There is a clear need to further study monocytes and how potential genetic risk is conferred through these cells in SSc. More detailed maps of chromatin architecture in monocytes are clearly needed. The emergence of genomic technologies such as Cleavage Under Targets and Release Using Nuclease (CUT\&RUN [41]), which allow DNA-protein interactions to be profiled on as few as 100,000 cells, now make the generation of such maps technically feasible. 
We note several limitations in this study. The first concerns our assessment of genetic influences on vascular function using data from HUVEC, which are an imperfect model. It is possible that if we were to examine endothelial cells from other tissues (e.g., dermal microvascular endothelial cells), we might obtain other results. Unfortunately, we were unable to find ChIPseq data from these cells on public databases. A second limitation is the fact that we focused our analyses on a limited number of risk loci, i.e., only those that have been rigorously replicated in a European population. A total of 34 regions have been identified in different studies in a broad range of populations [6], and we may therefore be underestimating genetic influences on immune cells, fibroblasts, or the endothelium, or overlooking genetic influences that may be exerted in non-European populations. We expanded our analysis to include these additional regions (33 regions studied as 1 failed to map) and found no significant enrichment in these regions $(p<0.01)$ for the functional marks that we queried. We note, however, that H3K27ac in monocytes, and H3K4me1 and H3K4me3 in HUVECs did achieve $p$ values $<0.05$. See expanded table in Additional file 4 . This difference in results from testing done with the 11 validated regions is expected as the added SNPs have not been confirmed by an independent study and some will likely fail in future validation studies. These findings illustrate the importance of being cautious regarding conclusions about genetic mechanisms based on regions that have not been validated.

Finally, we note that our investigations into TAD structure and the genes in $\mathrm{CD} 4+\mathrm{T}$ cells within them were generated from pediatric samples. There may be small differences between our findings and what would be seen in adult $\mathrm{CD} 4+\mathrm{T}$ cells, although recently published data demonstrate considerable conservation of the broader chromatin architecture across immune cells and immune cells lines [42].

\section{Conclusions}

The 11 replicated, non-HLA risk haplotypes for SSc are highly enriched for H3K4me1/H3K27ac-marked regulatory elements in a broad range of immune cells and fibroblasts. Furthermore, in CD4+ T cells, the risk haplotypes are parts of larger chromatin structures that include multiple genes that regulate a broad spectrum of immune processes relevant to SSc pathobiology. We were unable to find evidence for genetic influences on endothelial cell function, although replication of additional putative risk loci may reveal pathologically relevant genetic influences on these cells.

\section{Abbreviations}

GORILLA: Gene Ontology enRIchment anaLysis and visuaLizAtion; GWAS: Genome-wide association study; HLA: Human leukocyte antigen; HUVEC: Human umbilical vein endothelial cell; IPA: Ingenuity pathway analysis; LD: Linkage disequilibrium; REVIGO: Reduce visualize gene ontology; SNP: Single nucleotide polymorphism; SSc: Systemic sclerosis; TAD: Topologically associated domain.

\section{Supplementary Information}

The online version contains supplementary material available at https://doi. org/10.1186/s12920-021-00964-5.

\begin{abstract}
Additional file 1. UCSC Genome browser of chromatin landscape surrounding SSc-risk loci of interest. The yellow vertical line indicates position of the index SNP. Black horizontal bar at the top represents the haplotype block of the associated SSc-risk SNPs. The subsequent tracks as progress down are the bigWig files provided by Cistrome (hg38) for H3K27ac, H3K4me1, and H3K4me3 marks in B cells, fibroblasts, HUVECs, monocytes, and $T$ cells. Gene annotation set from GENCODE v32 is presented below histone tracks. Beneath that, the two rows of black vertical lines depict DNase hypersensitivity clusters in 95 cell types from ENCODE and transcription factor ChIP clusters of 340 factors from ENCODE. SNPedia SNPS are presented at the bottom.
\end{abstract}

Additional file 2. Significant biological process, molecular function, and cellular component GORILLA ontologies.

Additional file 3. Significant disease and biological function IPA annotations.

Additional file 4. Histone marks present in expanded sclerodermaassociated haplotypes.

\section{Acknowledgements}

Not applicable.

\section{Authors' contributions}

KEP, JNJ conceived and designed the study. KEP, JNJ analyzed the data and performed the statistical analyses. ET assisted in the analysis of the TADs in CD4+ T cells and monocytes. KEP, VMT, JNJ drafted the manuscript. KEP, VMT, $\mathrm{ET}$, and JNJ revised the manuscript and reviewed the final version. All authors read and approved the final manuscript.

\section{Funding}

This work was supported by R21-AR071878 from the National Institutes of Health, a Delivering on Discovery grant from the Arthritis Foundation, and an Innovative Research Grant from the Rheumatology Research Foundation.

\section{Availability of data and materials}

The datasets analyzed during the current study are available on the Cistrome database [http://cistrome.org/db/\#/] and Juicebox [http://aidenlab.org/juice box/].

\section{Declarations}

Ethics approval and consent to participate Not applicable.

Consent for publication

Not applicable.

\section{Competing interests}

KEP_None. VMT_-Principal investigator: National Science Foundation Award No. 1746694 and NIH NINDS award R43 NS115314-0. Awardee of the abovementioned Brain Aneurysm Foundation grant, Center for Advanced Technology grant, and Cummings Foundation grant. Co-founder: Neurovascular Diagnostics, Inc. ET_-Assistant Director at Quantitative Systems Pharmacology, Enhanced Pharmacodynamics, LLC, JNJ_Principal Investigator: R21-AR071878. 


\section{Author details}

${ }^{1}$ Canon Stroke and Vascular Research Center, Jacobs School of Medicine and Biomedical Sciences, University at Buffalo, Buffalo, NY, USA. ${ }^{2}$ Department of Neurosurgery, Jacobs School of Medicine and Biomedical Sciences, University at Buffalo, Buffalo, NY, USA. ${ }^{3}$ Department of Pathology and Anatomical Sciences, Jacobs School of Medicine and Biomedical Sciences, University at Buffalo, Buffalo, NY, USA. ${ }^{4}$ Department of Biomedical Engineering, University at Buffalo, Buffalo, NY, USA. ${ }^{5}$ Quantitative Systems Pharmacology, Enhanced Pharmacodynamics, LLC, Buffalo, NY, USA. ${ }^{6}$ Genetics, Genomics, and Bioinformatics Program, Jacobs School of Medicine and Biomedical Sciences, University at Buffalo, Buffalo, NY, USA. ${ }^{7}$ Department of Pediatrics, Jacobs School of Medicine and Biomedical Sciences, University at Buffalo, Buffalo, NY, USA.

Received: 5 November 2020 Accepted: 14 April 2021

Published online: 24 April 2021

\section{References}

1. Sobolewski P, Maślińska M, Wieczorek M, Łagun Z, Malewska A, Roszkiewicz M, et al. Systemic sclerosis-multidisciplinary disease: clinical features and treatment. Reumatologia. 2019;57(4):221-33.

2. Ho KT, Reveille JD. The clinical relevance of autoantibodies in scleroderma. Arthritis Res Ther. 2003:5(2):80-93.

3. Sunderkötter C, Riemekasten G. Pathophysiology and clinical consequences of Raynaud's phenomenon related to systemic sclerosis. Rheumatology. 2006;45(suppl_3):iii33-5.

4. Walker UA, Tyndall A, Czirják L, Denton C, Farge-Bancel D, Kowal-Bielecka $\mathrm{O}$, et al. Clinical risk assessment of organ manifestations in systemic sclerosis: a report from the EULAR Scleroderma Trials And Research group database. Ann Rheum Dis. 2007;66(6):754.

5. Matucci-Cerinic M, Kahaleh B, Wigley FM. Review: evidence that systemic sclerosis is a vascular disease. Arthritis Rheum. 2013;65(8):1953-62.

6. Pattanaik D, Brown M, Postlethwaite BC, Postlethwaite AE. Pathogenesis of systemic sclerosis. Front Immunol. 2015;6:272.

7. Gladman DD, Kung TN, Siannis F, Pellett F, Farewell VT, Lee P. HLA markers for susceptibility and expression in scleroderma. J Rheumatol. 2005;32(8):1481.

8. Korman BD, Criswell LA. Recent advances in the genetics of systemic sclerosis: toward biological and clinical significance. Curr Rheumatol Rep. 2015;17(3):21

9. Zhou X, Lee JE, Arnett FC, Xiong M, Park MY, Yoo YK, et al. HLA-DPB1 and DPB2 are genetic loci for systemic sclerosis: a genome-wide association study in Koreans with replication in North Americans. Arthritis Rheum. 2009;60(12):3807-14.

10. Agarwal S, Tan F, Arnett F. Genetics and genomic studies in scleroderma (systemic sclerosis). Rheum Dis Clin N Am. 2008;34(1):17.

11. Arnett FC, Gourh P, Shete S, Ahn CW, Honey RE, Agarwal SK, et al. Major histocompatibility complex (MHC) class II alleles, haplotypes and epitopes which confer susceptibility or protection in systemic sclerosis: analyses in 1300 Caucasian, African-American and Hispanic cases and 1000 controls. Ann Rheum Dis. 2010;69(5):822.

12. Radstake TRDJ, Gorlova O, Rueda B, Martin J-E, Alizadeh BZ, Palomino-Morales R, et al. Genome-wide association study of systemic sclerosis identifies CD247 as a new susceptibility locus. Nat Genet. 2010;42(5):426-9.

13. Jiang K, Zhu L, Buck MJ, Chen Y, Carrier B, Liu T, et al. Disease-associated SNPs from non-coding regions in Juvenile idiopathic arthritis are located within or adjacent to functional genomic elements of human neutrophils and CD4+ T Cells. Arthritis Rheumatol (Hoboken, NJ). 2015;67(7):1966-77.

14. Hui-Yuen JS, Zhu L, Wong LP, Jiang K, Chen Y, Liu T, et al. Chromatin landscapes and genetic risk in systemic lupus. Arthritis Res Ther. 2016;18(1):281.

15. Poppenberg KE, Jiang K, Tso MK, Snyder KV, Siddiqui AH, Kolega J, et al. Epigenetic landscapes suggest that genetic risk for intracranial aneurysm operates on the endothelium. BMC Med Genom. 2019;12(1):149.

16. Farh KK-H, Marson A, Zhu J, Kleinewietfeld M, Housley WJ, Beik S, et al. Genetic and epigenetic fine mapping of causal autoimmune disease variants. Nature. 2015;518(7539):337-43.
17. Pelikan RC, Kelly JA, Fu Y, Lareau CA, Tessneer KL, Wiley GB, et al. Enhancer histone-QTLs are enriched on autoimmune risk haplotypes and influence gene expression within chromatin networks. Nat Commun. 2018;9(1):2905.

18. Arnold M, Raffler J, Pfeufer A, Suhre K, Kastenmüller G. SNiPA: an interactive, genetic variant-centered annotation browser. Bioinformatics. 2014;31(8):1334-6.

19. Jiang K, Zhu L, Buck MJ, Chen Y, Carrier B, Liu T, et al. Disease-associated SNPs from non-coding regions in juvenile idiopathic arthritis are located within or adjacent to functional genomic elements of human neutrophils and CD4+ T cells. Arthritis Rheumatol. 2015;67(7):1966-77.

20. Hnisz D, Day DS, Young RA. Insulated neighborhoods: structural and functional units of mammalian gene control. Cell. 2016;167(5):1188-200.

21. Kessler $\mathrm{H}$, Jiang $\mathrm{K}$, Jarvis JN. Using chromatin architecture to understand the genetics and transcriptomics of juvenile idiopathic arthritis. Front Immunol. 2018;9:2964.

22. Gasperini M, Hill AJ, McFaline-Figueroa JL, Martin B, Kim S, Zhang MD, et al. A genome-wide framework for mapping gene regulation via cellular genetic screens. Cell. 2019;176(1):377-90.e19.

23. Tarbell EJK, Hennon TR, Holmes L, Williams S, Fu Y, Gaffney PM, Liu T, Jarvis JN. CD4+ T cells from children with active juvenile idiopathic arthritis show altered chromatin features associated with transcriptional abnormalities. Sci Rep. 2021 (in press).

24. Durand NC, Robinson JT, Shamim MS, Machol I, Mesirov JP, Lander ES, et al. Juicebox provides a visualization system for $\mathrm{Hi}-\mathrm{C}$ contact maps with unlimited zoom. Cell Syst. 2016;3(1):99-101.

25. Phanstiel DH, Van Bortle K, Spacek D, Hess GT, Shamim MS, Machol I, et al. Static and dynamic DNA loops form AP-1 bound activation hubs during macrophage development. bioRxiv. 2017:142026.

26. Eden E, Navon R, Steinfeld I, Lipson D, Yakhini Z. GOrilla: a tool for discovery and visualization of enriched $\mathrm{GO}$ terms in ranked gene lists. BMC Bioinform. 2009;10:48.

27. Supek F, Bošnjak M, Škunca N, Šmuc T. REVIGO summarizes and visualizes long lists of gene ontology terms. PLoS ONE. 2011;6(7):e21800-e.

28. Krämer A, Green J, Pollard J Jr, Tugendreich S. Causal analysis approaches in ingenuity pathway analysis. Bioinformatics (Oxford, England). 2014;30(4):523-30.

29. Maurano MT, Humbert R, Rynes E, Thurman RE, Haugen E, Wang H, et al. Systematic localization of common disease-associated variation in regulatory DNA. Science (New York, NY). 2012;337(6099):1190-5.

30. Maria ATJ, Partouche L, Goulabchand R, Rivière S, Rozier P, Bourgier C, et al. Intriguing relationships between cancer and systemic sclerosis: role of the immune system and other contributors. Front Immunol. 2019:9:3112.

31. Dolcino M, Pelosi A, Fiore PF, Patuzzo G, Tinazzi E, Lunardi C, et al. Gene profiling in patients with systemic sclerosis reveals the presence of oncogenic gene signatures. Front Immunol. 2018;9:449.

32. Grassi W, Medico PD, Izzo F, Cervini C. Microvascular involvement in systemic sclerosis: capillaroscopic findings. Semin Arthritis Rheum. 2001;30(6):397-402.

33. Fleischmajer R, Perlish JS. Capillary alterations in scleroderma. J Am Acad Dermatol. 1980;2(2):161-70.

34. Postlethwaite AE, Shigemitsu H, Kanangat S. Cellular origins of fibroblasts: possible implications for organ fibrosis in systemic sclerosis. Curr Opin Rheumatol. 2004;16(6):733-8.

35. Ginhoux F, Jung S. Monocytes and macrophages: developmental pathways and tissue homeostasis. Nat Rev Immunol. 2014;14:392-404 (1474-1741 (Electronic)).

36. Katebi M, Fernandez P, Chan ESL, Cronstein BN. Adenosine A2A receptor blockade or deletion diminishes fibrocyte accumulation in the skin in a murine model of scleroderma, bleomycin-induced fibrosis. Inflammation. 2008;31(5):299-303.

37. Quan TE, Cowper S, Wu S-P, Bockenstedt LK, Bucala R. Circulating fibrocytes: collagen-secreting cells of the peripheral blood. Int J Biochem Cell Biol. 2004;36(4):598-606.

38. Mathai SK, Gulati M, Peng X, Russell TR, Shaw AC, Rubinowitz AN, et al. Circulating monocytes from systemic sclerosis patients with interstitial lung disease show an enhanced profibrotic phenotype. Lab Investig. 2010;90(6):812-23.

39. Scott MKD, Quinn K, Li Q, Carroll R, Warsinske H, Vallania F, et al. Increased monocyte count as a cellular biomarker for poor outcomes in fibrotic 
diseases: a retrospective, multicentre cohort study. Lancet Respir Med. 2019;7(6):497-508.

40. Tadmor T, Bari A, Marcheselli L, Sacchi S, Aviv A, Baldini L, et al. Absolute monocyte count and lymphocyte-monocyte ratio predict outcome in nodular sclerosis Hodgkin lymphoma: evaluation based on data from 1450 patients. Mayo Clin Proc. 2015;90(6):756-64.

41. Skene PJ, Henikoff S. An efficient targeted nuclease strategy for highresolution mapping of DNA binding sites. Elife. 2017;6:e21856.
42. Zhang S, Chen F, Bahar I. Differences in the intrinsic spatial dynamics of the chromatin contribute to cell differentiation. Nucleic Acids Res. 2020;48(3):1131-45.

\section{Publisher's Note}

Springer Nature remains neutral with regard to jurisdictional claims in published maps and institutional affiliations.
Ready to submit your research? Choose BMC and benefit from:

- fast, convenient online submission

- thorough peer review by experienced researchers in your field

- rapid publication on acceptance

- support for research data, including large and complex data types

- gold Open Access which fosters wider collaboration and increased citations

- maximum visibility for your research: over $100 \mathrm{M}$ website views per year

At BMC, research is always in progress.

Learn more biomedcentral.com/submissions 